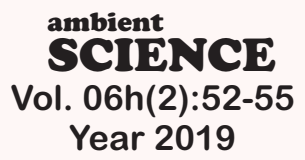

\title{
Anthropometric Analysis of Nasal Profile in Rhinoplasty Candidates from Southwest Iran
}

\section{Kamran Babaei ${ }^{1}$, Abdolazim Ghalambor ${ }^{1}$, Mohamad Ali Paidar ${ }^{*}$,Hamid Reza Abbasi Harofteh$^{2}$, Mehran Karvar ${ }^{3}$}

${ }^{1}$ Department of Plastic and Reconstructive Surgery, School of Medicine, Ahvaz Jundishapur University of Medical Sciences, Ahvaz, Iran

${ }^{2}$ Department of Anesthesiology, School of Medicine, Shahid Sadoughi University of Medical Sciences, Yazd, Iran

${ }^{3}$ School of Medicine, KermanUniversity of Medical Sciences, Kerman, Iran

Study Area:Ahvaz, Iran

Coordinates: $31^{\circ} 19^{\prime} 13^{\prime \prime N}$; $48^{\circ} 40^{\prime} 09^{\prime E}$

Key words: Facial beauty, Plastic Surgery, Morphometric measurements.

Ethical Code: Approved by the ethics committee of the Ahvaz Jundishapur University of Medical Sciences (Code of Ethics: IR.AJUMS.REC.1396.217).

\section{Introduction:}

Rhinoplasty is one of the most common facial plastic surgeries the outcomes of which can affect both the shape and function of the nose. Due to the anatomical position of the nose and its centrality in the face, obtaining the desired outcome in rhinoplasty is an important consideration for both, patients and the surgeon (Doddi \& Eccles, 2010). In rhinoplasty, the difference between desirable and undesirable outcomes varies within a few millimeters. To obtain optimum outcomes, a comprehensive examination of the structure, anatomy, and function of the nose before surgery is essential. The shape, size, and proportion of the nose play an important role in nasal beauty. Nasal indices and parameters such as length, height, proportion, and angles are of crucial importance in anthropometry (Lines et al., 1978; Farkas, 1994; Aiach et al., 1996; Farkas et al., 200o; Fontana \& Muti, 2001; Aung et al., 2007).

Previous studies have already reported that race, culture, and ethnicity affect the expectation and perception of an individual on the definition of nasal beauty (IglesiasLinares et al., 2011). Several studies have been conducted on nasal anthropometric indices in different races and ethnic

\section{Abstract}

Race, culture, and ethnicity affect the anatomy and morphology of human nose. Moreover, expectation and perception of an individual on nasal beauty mainly differ between different racial and ethnic groups. This study aims to evaluate nasal anthropometric indices of candidates of rhinoplasty based on a retrospective hospital record. The demographic information and clinical data of the subjects and the surgical outcomes were extracted from the hospital records. Moreover, the pre- and post-plastic surgery images were used to calculate different nasal anthropometric indices. Data of 104 (age range: 17-54 years old) candidates of rhinoplasty were analyzed. The major ethnic was Arabians (39.4\%). The mean nasal length and height in males were statistically lower than the females. No significant difference was observed between the two groups in terms of other indices. The average age group of rhinoplasty candidates was found to be very low in Iranian society. As per our findings, specific charts could be developed for the anthropometric analysis of the patient's face and body. Furthermore, a general outline of the nose could be developed for future rhinoplasty to achieve better postoperative outcomes.

groups (Farkas et al., 1998; Uzun et al., 2006; Heidari et al., 2009; Al-Qattan et al., 2012; Farkas et al., 2014; Packiriswamy et al., 2016).Therefore, defining objectives and subjective standard measures of nasal beauty for each patient is important to know for plastic surgeons (Doddi \& Eccles, 2010). Iranian patients constitute one of the largest group of candidates for rhinoplasty in the world. However, there are no suitable criteria are in use for anthropometric analysis of the pre- and post-surgery images of Iranian patients. Thus, the present study aims to evaluate nasal anthropometric indices of rhinoplasty candidates in a major hospital in southwest Iran. The findings of this study could be used to develop a set of objective and subjective indices for anthropometric analyses of the pre- and postsurgery images in Iranian patients.

\section{Methodology:}

This was a retrospective study conducted on the medical records of rhinoplasty candidates referred to the plastic surgery department of Taleghani Hospital, Ahvaz, Iran between 2014 and 2016. The demographic information, clinical data of the patients and the surgical outcomes were 
extracted from the hospital records. Further, the pre- and post-plastic surgery images were used to calculate different nasal anthropometric indices.

Exclusion criteria were deformity of the craniofacial or congenital facial, history of surgery or trauma in the maxillofacial area, nasal congestion, rhinitis, or other allergic diseases, skin disease in the nasolabial area, abnormal occlusion, hypergenesis or hypogenesis in the middle and lower thirds of the face, obvious asymmetry (length, width, height) in ears, eyes, nose, lip, and teeth loss that can interfere with the height in lower third of the face.

After determining the sample, based on the information derived from patients' profiles and their images, using Vernier caliper, steel graded ruler and goniometers, nasal indices including 12 linear distances with an accuracy of $0.1 \mathrm{~mm}$ and 5 angles were measured. Patients photographs have been obtained in frontal, left lateral, and base views in a normal anatomic position.

We used the Farkas guideline (Farkas, 1994) to measure anthropometric indices for all patients and the measurement points wereset as nasion (n); subnasale (sn); pronasale (prn); alar curvature (ac).

Measured indices were: nasal length (nasion to pronasale); nasal height (nasion to subnasale); alar width; nasofrontal angle; nasolabial angle (the angle between the columella and the upper lip); nasal tip angle (the angle between the line that passes through dorsum of the nose with the line passing throughcolumella); nasofasial angle (angle between the line that passes through dorsum of the nose with the line passing through Gelabela and Pugunion); nasal index (nose width/ nose height $\times 100$ ); nasal width (from right to left nasal alae); and nasal depth. SPSS (Version 22) using Student T-test and Chi-square test were used to compare nasal indices data.

\section{Result:}

The demographic information of the studied population is presented in Table-1. The majority of patients were Arabs (39.4\%) and mainly females (73.1\%). Moreover, the majority of the rhinoplasty candidates were single ( $58.7 \%)$. The age range of the patients was 17 to 54 years old..

Table-1: Demographic Characteristics of the subjects in groups

\begin{tabular}{lll}
\hline Variables & Parameters & Frequencies (\%) \\
\hline Race & Arab & $41(39.4)$ \\
& Fars & $37(35.6)$ \\
& Bakhtiari & $12(11.5)$ \\
& Other & $14(13.5)$ \\
Gender & Male & $28(26.9)$ \\
Marital Status & Female & $76(73.1)$ \\
& Married & $61(58.7)$ \\
Age range (year) & Single & $43(41.3)$ \\
& o-20 & $24(23.1)$ \\
& $21-30$ & $56(53.8)$ \\
& $31-40$ & $19(18.3)$ \\
& $>40$ & $5(4.8)$ \\
\hline
\end{tabular}

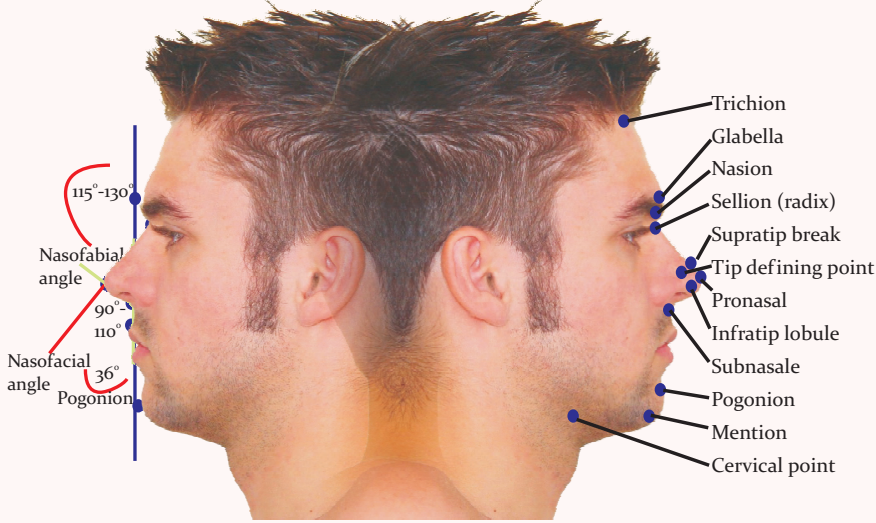

Plate-1: Measurements and facial terms used in the article

A total of $17(65.38 \%)$ patients reported as complete satisfaction with nipple-areola feeling, 8 (30.77\%) patients expressed nipple-areola feeling compared to pre-operative as "moderate", and the nipple-areola feeling was weak in 1 (3.85\%) patient. Only one patient was dissatisfied with the nipple-areola feeling. The distance between sternal notch to the nipple and the distance between the IMF to the inferior nipple after the operation were evaluated in the last followup, the results of which are shown in Table-2.

\section{Discussion:}

The overall understanding of the nasal structure in groups of people with specific ethnicity, culture, and genetics is crucial to evaluate rhinoplasty candidates before and after surgery. Therefore, an anthropometric study of the nose allows the surgeon to consider the candidate's perception of a beautiful nose in accordance with different racial and cultural features (Doddi \& Eccles, 2010; Farkas et al., 2000; Farkas, 1994; Iglesias-Linares et al., 2011). How far the taste is not in conflict with the candidate's nasal health, the surgeon can design a comprehensive and pre-planned scheme for each patient in accordance with the aesthetic principles. There are many studies on nasal anthropometric indices of different races around the world. Farkas (1994) presented an overview of the anthropometry of an ideal face, but their study was based on the North American population and therefore cannot be generalized to all groups and races. However, so far few studies have been conducted in Iran on nasal anthropometric features for different patients groups. Heidari et al. (2009) investigated the anthropometric indices in women candidates for rhinoplasty and compared the measures between the two ethnicities of Baluchi and Sistani of Southeastern Iran. They reported that despite a significant difference in terms of nasal dimension, the prevalent types of nose and face were identical in both groups. Sepehr et al. (2012) compared anthropometric measures of Persian woman face and nose with the North American white women. They concluded that anthropometric differences between Persian women and North American White women reflect fundamental differences in the osseochondrous scaffold and soft tissue 
Table 2. Anthropometric measures of nose of study patients

\begin{tabular}{|c|c|c|c|c|c|c|}
\hline \multirow[t]{2}{*}{ Variables } & \multicolumn{2}{|c|}{ Female $(n=76)$} & \multicolumn{2}{|c|}{ Male $(n=28)$} & \multirow{2}{*}{$\begin{array}{l}\text { Total }(n=104) \\
\text { Mean } \pm \text { SD }\end{array}$} & \multirow[t]{2}{*}{ P-Value } \\
\hline & Mean \pm SD & Min-max & Mean \pm SD & Min-max & & \\
\hline Nose length (n-prn) & $6.687 \pm 0.498$ & $4 \cdot 9-7 \cdot 5$ & $6.646 \pm 0.399$ & $5 \cdot 9-7 \cdot 4$ & $6.676 \pm 0.472$ & 0.000 \\
\hline Nose height (n-sn) & $6.376 \pm 0.440$ & $5 \cdot 0-7 \cdot 4$ & $6.361 \pm 0.343$ & $5.9-7.2$ & $6.372 \pm 0.415$ & 0.005 \\
\hline Nose depth (sn-prn) & $4.031 \pm 0.501$ & $3.2-6.6$ & $4.102 \pm 0.397$ & $3.1-4.75$ & $4.050 \pm 0.475$ & 0.972 \\
\hline Nose width (alr-all) & $3.645 \pm 0.391$ & $3 \cdot 0-4 \cdot 5$ & $3.696 \pm 0.411$ & $3.1-4.7$ & $3.659 \pm 0.395$ & 0.415 \\
\hline Width nose anatomical & $3.421 \pm 0.396$ & $2 \cdot 7-4 \cdot 9$ & $3.496 \pm 0.385$ & $3 \cdot 0-4 \cdot 6$ & $3.441 \pm 0.393$ & 0.068 \\
\hline Nasofrontal angle & $127.316 \pm 6.729$ & $107.0-141.0$ & $130.107 \pm 6.696$ & $115.0-140.0$ & $128.067 \pm 6.802$ & 0.646 \\
\hline Nasolabial angle & $92.223 \pm 6.662$ & 76.00-106.00 & $91.321 \pm 6.122$ & $77.0-101.0$ & $91.981 \pm 6.505$ & 0.949 \\
\hline Nasal tip angle & $66.408 \pm 5.607$ & $58.0-87.0$ & $67.107 \pm 6.613$ & $61.0-87.0$ & $66.596 \pm 5.870$ & 0.909 \\
\hline Nasofacial & $37.039 \pm 4.431$ & $27 \cdot 0-45 \cdot 0$ & $37.036 \pm 4.05$ & $30.0-45 \cdot 0$ & $37.039 \pm 4.313$ & 0.220 \\
\hline Index & $1.662 \pm 0.187$ & $1.02-2.28$ & $1.635 \pm 0.187$ & $1.33-2.30$ & $1.655 \pm 0.187$ & 0.128 \\
\hline
\end{tabular}

covering of the face. The findings of the study by Moshkelgosha et al. (2015) conducted on adolescent Persian population demonstrated that Persian boys have bigger noses (in terms of nasal length, depth, and prominence) than Persian girls. They reported that the height of the nasal tip did not show sexual variations.

The findings of this study on anthropometric nasal indices on 104 patients who underwent rhinoplasty in the Taleghani Hospital, Ahvaz, Iran provide useful implications. Compared with the findings of the Farkas (1994) and Farkas et al. (2005), the nasal length, height, width, and nasofacial angle values in our study population were higher than North American white women. Nasolabial and nasofrontal angles were larger in North American white women than the Ahvaz rhinoplasty candidates. These differences could be the result of variation in races and ethnicity. The mean nasal length, width, and height in our study population were higher than the values reported by Heidari et al. (2009). The mean values of nasal length, depth, and width in our Persian sample was bigger, but the mean nasofrontal and nasolabial angles were smaller than those reported by Moshkelgosha et al. (2015).

Our study revealed that the age for rhinoplasty requirement in Ahvaz society was low and the majority of patients were under the age of 30 years old. Considering the possible complications of any type of surgery, including cosmetic surgery, even years after the surgery, we will witness a flow of candidates for re-surgical procedures resulting from previous rhinoplasties in near future, which should be considered in terms of its huge financial burden on patients and its health implications. The increasingly growing demand for rhinoplasty among single individuals compared with the married individuals could be attributed to and discussed on specific cultural aspects in the Iranian community. The interest and demand for rhinoplasty surgeries among females are significantly higher than the males in the Iranian community thus it is estimated that women constitute three-quarters of the rhinoplasty candidates. Most candidates are unhappy with the dorsal hump or the size, shape, and width of their nose, desired to correct this misalignment. Among them, the large nasal size was one of the most common complaints and the main reason for rhinoplasty surgery. A crucial point in the anthropometric study of patients was the nasofrontal angle, the mean of which varied between men and women. It can have several reasons, including low radix and tip drop, each of which requires a preoperative examination and assessment to obtain a solution during the surgery. Our findings established the possibility to draw up charts based on nasal and facial anthropometric analysis of the patient so that this chart is completed before the surgery and the outcome is presented to the candidate. It can offer a general overview and outline of the postoperative shape of the nose, and if approved by the candidate, the surgeon can perform the surgery. In this way, there would be fewer issues between the surgeon and patients with regard to postoperative outcomes. The reliability of results is increased when a larger sample size would be studied. Considering that this study was conducted in a public and educational center and the candidates were mainly from a specific cultural and economic background, it seems that a study at a wider scale that covers candidates in both private and public clinics could provide more reliable results.

Conclusively, we found that most of the candidates of rhinoplasty in Iranian society are the youths. Anthropometric measures of the nose are unique to each individual and they are proportional to other components of the face. Our study showed that specific charts could be developed for the anthropometric analysis of patients' face and body. This chart can be presented to the candidates to provide them an overview and outline of the nose after the surgery, and if the candidate expresses satisfaction, the surgeon can perform the surgery. It also helps resolve the issues that may arise between the surgeon and patient regarding the postoperative outcomes. Furthermore, a general outline of the nose could be developed for the future rhinoplasty that helps patient and surgeon achieve better postoperative outcomes.

\section{Acknowledgment:}

This study was approved in Ahvaz Jundishapur University of

Medical Sciences (AJUMS). The study was $f$ inancially supported byAJUMS (Grant.No.:U-96046). 


\section{ORIGINAL ARTICLE}

\section{References:}

Aiach, G. (1996): Rhinoplasty, p. 171-198. In: Marchac D, Granick MS, Solomon MP, (eds.) Male Aesthetic Surgery. Pub. by: Butterworths-Heinemann, Newton.

Al-Qattan, M.M., Alsaeed, A.A., Al-Madani, O.K., Al-Amri, N.A. \& Al-Dahian, N.A. (2012): Anthropometry of the Saudi Arabian nose. J. Craniofac. Surg., 23(3):821-824.

Aung, S.C., Foo, C.L. \& Lee, S.T. (2000): Three dimensional laser scan assessment of the oriental nose with a new classification of oriental nasal types. Br. J. Plast. Surg., 53(2):109-116.

Doddi, N.M. \& Eccles, R. (2010): The role of anthropometric measurements in nasal surgery and research: a systematic review. Clin.Otolaryngol., 35(4):277-283.

Farkas, L.G. (1994): Anthropometry of the Head and Face. Pub. by: Raven Press, New York, US. P. 405.

Farkas, L.G., Forrest, C.R. \& Phillips, J.H. (2000): Comparison of the morphology of the "cleft face" and the normal face: defining the anthropometric differences. L. Craniofac. Surg., 11(2):76-82.

Farkas, L.G., Katic, M.J. \& Forrest, C.R., Alt, K.W., Bagic, I., Baltadjiev, G., Cunha, E., Cvicelová, M., Davies, S., Erasmus, I., Gillett-Netting, R., Hajnis, K., Kemkes-Grottenthaler, A., Khomyakova, I., Kumi, A., Kgamphe, J.S., Kayo-daigo, N., Le, T., Malinowski, A., Negasheva, M., Manolis, S., Ogetürk, M., Parvizrad, R., Rösing, F., Sahu, P., Sforza, C., Sivkov, S., Sultanova, N., Tomazo-Ravnik, T., Tóth, G., Uzun, A. \& Yahia, E. (2005): International anthropometric study of facial morphology in various ethnic groups/races.J. Craniofac. Surg., 16(4):615-646.

Farkas, L.G., Phillips, J.H. \& Katic, M. (1998): Anthropometricanatomical and morphological nose widths in CanadianCaucasian adults. Can.J. Plast. Surg., 6(3):149-151.
Ambient Science, 2019: Vol. 06h(2); 52-55 DOI:10.21276/ambi.2019.06h.2.oa12

Fontana, A.M. \& Muti, E. (2001): Our “idees fixes" in rhinoplasty: the nasofrontalangle and the alar lateral crus. Aesth. Plast. Surg., 25(1):1-7.

Heidari, Z., Mahmoudzadeh-Sagheb, H., Khammar, T. \& Khammar, M. (2009): Anthropometric measurements of the external nose in 18-25-year-old Sistani and Baluch aborigine women in the southeast of Iran. Folia Morphol. (Warsz), 68(2):88-92.

Iglesias-Linares, A., Yanez-Vico, R.M., Moreno-Manteca, B., Moreno-Fernández, A.M., Mendoza-Mendoza, A. \& SolanoReina, E. (2011): Common standards in facial esthetics: craniofacial analysis of most attractive black and white subjects according to people magazine during previous 10 years. J. Oral Macillofac. Surg., 69(6):e216-224J.

Lines, P., Lines, R. \& Lines, C.(1978): Profilometrics and facial aesthetics.Am. J. Orthod., 73(6):648-657.

Moshkelgosha, V., Fathinejad, S., Pakizeh, Z., Shamsa, M. \& Golkari, A. (2015): Photographic facial soft tissue analysis by means of linear and angular measurements in an adolescent Persian population. Open Dent. J., 9:346-356.

Packiriswamy, V., Bashour, M. \& Nayak, S. (2016): Anthropometric analysis of the South Indian woman's nose. Facial Plast. Surg., 32(3):304-308.

Sepehr, A., Mathew, P.J., Pepper, J.P., Karimi, K., Devcic, Z. \& Karam, A.M. (2012): The Persian woman's face: a photogrammetric analysis. Aesthetic Plast. Surg., 36(3):687691.

Uzun, A., Akbas, H., Bilgic, S., Emirzeoglu, M., Bostanc, O., Sahin, B. \& Bek, Y. (2006): The average values of the nasal anthropometric measurements in 108 young Turkish males. Auris Nasus Larynx, 33(1):31-35. 